\title{
Sleep and Performance
}

Editors

RACHEL R. MARKWALD

ANNE GERMAIN

\section{SLEEP MEDICINE CLINICS}

www.sleep.theclinics.com

Consulting Editor

TEOFILO LEE-CHIONG Jr

March 2020 • Volume 15 • Number 1 